\title{
Ventilação mecânica, parâmetros de troca gasosa e desmame do ventilador em pacientes com COVID-19
}

\author{
Mechanical ventilation settings, gas exchange parameters and ventilator weaning in \\ patients with COVID-19
}
Ajustes de ventilación mecánica, parámetros de intercambio de gases y destete del ventilador en pacientes con COVID-19

Letícia Lourenço Botelho ${ }^{*}$, Álisson Francisco Colombi Sebim², Ana Clara Abreu Lima de Paula ${ }^{3}$, Anne de Morais Gomes ${ }^{2}$, Idemar Luís Cover Filho ${ }^{4}$, Juliana Rocha Santos ${ }^{2}$ Maria Luiza Breijão da

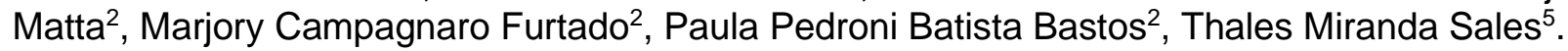

\section{RESUMO}

Objetivo: Descrever e discutir os parâmetros do suporte ventilatório de pacientes com COVID-19, assim como os critérios e métodos utilizados para o desmame da Ventilação Mecânica Invasiva (VMI). Revisão bibliográfica: De acordo com a análise literária, o modo ventilação com pressão controlada com volume corrente baixo está dentro dos parâmetros iniciais recomendados para a VMI. O desmame, por sua vez, engloba fatores individuais, como a carga e a capacidade do sistema respiratório, e os aspectos psicológicos, físicos e nutricionais. Em pacientes com COVID-19, os critérios tradicionais de extubação permanecem os mesmos, sendo o processo feito de forma gradual, englobando a redução gradativa do nível de Pressão Expiratória Final Positiva (PEEP) ao mesmo tempo em que é feita a checagem das metas de oxigenação. Além disso, a fisioterapia respiratória se faz necessária antes e após a realização da extubação. Considerações finais: Apesar da escassez de estudos e a falta de consenso em relação às condutas no manejo da COVID-19, estabeleceu-se um ponto de partida efetivo no tratamento de tais pacientes no que diz respeito aos parâmetros da VMI e ao desmame de tal terapia, culminando no sucesso de tais intervenções.

Palavras-chave: Respiração artificial, Desmame do respirador, Infecções por coronavírus.

\section{ABSTRACT}

Objetive: Describe and discuss the parameters of ventilatory support of patients with COVID-19, as well as the criteria and methods used for weaning from Invasive Mechanical Ventilation (IMV). Bibliographic review: According to the literary analysis, the controlled pressure ventilation mode with low tidal volume is within the recommended initial parameters for IMV. Weaning, in turn, includes individual factors, such as the load and capacity of the respiratory system, and the psychological, physical and nutritional aspects. In patients with COVID-19, the traditional extubation criteria remain the same, the process being done gradually, encompassing the gradual reduction of the Positive End Expiratory Pressure (PEEP) level while checking the oxygenation targets. In addition, respiratory physiotherapy is necessary before and after extubation. Final considerations: Despite the scarcity of studies and the lack of consensus regarding the management of COVID-19, an effective starting point has been established in the treatment of such patients with regard to IMV parameters and the weaning of such therapy, culminating in the success of such interventions.

Key words: Respiration artificial, Ventilator weaning, Coronavirus infections.

\footnotetext{
${ }^{1}$ Centro Universitário de Valença (UNIFAA), Valença - RJ. *E-mail: leticia_botellho@hotmail.com

2 Universidade Vila Velha (UVV), Vila Velha - ES.

${ }^{3}$ Universidade Federal de Juiz de Fora (UFJF), Juiz de Fora - MG.

${ }^{4}$ Faculdade de Ciências Médicas da Paraíba (FCMPB), Cabedelo - PB.

${ }_{5}^{5}$ Universidade Federal de Ouro Preto (UfOP), Ouro Preto - MG.
} 


\section{RESUMEN}

Objetivo: Describir y discutir los parámetros de soporte ventilatorio de los pacientes con COVID-19, así como los criterios y métodos utilizados para el destete de la Ventilación Mecánica Invasiva (VMI). Revisión bibliográfica: Según el análisis literario, el modo de ventilación a presión controlada con volumen corriente bajo se encuentra dentro de los parámetros iniciales recomendados para VMI. El destete, a su vez, incluye factores individuales, como la carga y capacidad del sistema respiratorio, y los aspectos psicológicos, físicos y nutricionales. En pacientes con COVID-19, los criterios de extubación tradicionales siguen siendo los mismos, el proceso se realiza de forma gradual, abarcando la reducción gradual del nivel de Presión Espiratoria Final Positiva (PEEP) mientras se controlan los objetivos de oxigenación. Además, la fisioterapia respiratoria es necesaria antes y después de la extubación. Considerações finales: A pesar de la escasez de estudios y la falta de consenso en relación a la conducta en el manejo del COVID-19, se ha establecido un punto de partida efectivo en el tratamiento de dichos pacientes en cuanto a los parámetros de la VMI y el destete de dicha terapia. culminando en el éxito de tales intervenciones.

Palabras clave: Respiración artificial, Desconexión del ventilador, Infecciones por coronavirus.

\section{INTRODUÇÃO}

No final de 2019 surgiu em Wuhan, na China, uma nova cepa viral denominada coronavírus da Síndrome Respiratória Aguda Grave 2 (SARS-CoV-2), que se espalhou mundialmente e, em 11 de março de 2020 foi considerada como uma pandemia pela Organização Mundial da Saúde (OMS) (BASTOS GAN, et al., 2020). Até o final do mês de 2021 foram registrados, mundialmente, cerca de 170 milhões de casos confirmados e 3,5 milhões óbitos pela doença do coronavírus 2019 (COVID-19) (OMS, 2021).

Ainda sem definição fisiopatológica, estudos têm mostrado que a COVID-19 é capaz de modificar a Enzima Conversora de Angiotensina 2 (ECA2), produzida nos pulmões, tornando este órgão altamente suscetível ao vírus (AYRES J, 2020). A doença causada pela COVID-19 apresenta amplo espectro clínico, que varia desde assintomática até uma Síndrome do Desconforto Respiratório Agudo (SDRA), necessitando de internação em Unidade de Terapia Intensiva (UTI). A forma grave da doença foi registrada em $14 \%$ dos casos e a falência respiratória com necessidade de ventilação mecânica, em $5 \%$, de acordo com dados do Chinese Center for Disease Control and Prevention, com uma amostra de 44.500 pessoas com diagnóstico confirmado de COVID-19 (ARAÚJO MS, et al., 2021; CARRILLO-ESPER R, et al., 2020).

A admissão na UTI está comumente ligada à falência respiratória aguda, que pode ser considerada como a presença de hipoxemia, falência ventilatória, ou ambas, e necessidade de intervenções ventilatórias de suporte, tanto invasiva quanto não invasiva. Nos casos mais leves é utilizado o tratamento de suporte não invasivo, o qual fornece interfaces externas como máscaras nasais, faciais, bocais e oronasal, com objetivo de minimizar o esforço respiratório e melhorar as trocas gasosas (LEWIS SR, et al., 2021).

Já a ventilação mecânica invasiva, utilizada nos casos mais graves, visa corrigir a hipoxemia e hipercapnia, sendo necessária a utilização de um ventilador mecânico, em sincronia com o paciente, através de diversos modos ventilatórios, sendo eles: Ventilação com Volume Controlado (VCV), Ventilação com Pressão Controlada (PCV), Ventilação com Pressão de Suporte (PSV), pressão positiva contínua nas vias aéreas e ventilação mandatória intermitente sincronizada (NOBRE KMF, 2020). Nos pacientes atingidos pela SDRA causada pela COVID-19 foi observada uma baixa complacência pulmonar, sendo usada uma ventilação protetora, em especial com relação ao uso de um volume corrente mais baixo e a posição prona como tratamento para hipoxemia refratária (BOTTA M, et al., 2021).

Por fim, o desmame envolve a redução gradual dos parâmetros ventilatórios ao mesmo tempo em que aborda aspectos médicos, psicológicos, físicos e nutricionais do atendimento ao paciente. É um processo individualizado e que deve ser feito de forma gradual, reduzindo gradativamente os níveis de Pressão Expiratória Final Positiva (PEEP) de acordo com as metas de oxigenação que vão sendo alcançadas ao longo do processo de despressurização do sistema ventilatório. É necessária prudência ao retirar o ventilador, uma 
vez que a retirada precoce da ventilação mecânica pode levar a pior prognóstico do paciente (OVADYA D, et al., 2020; CARRILLO-ESPER R, et al., 2020).

Assim, mesmo considerando que as condutas têm evoluído de forma célere, o objetivo do presente estudo consiste em descrever os parâmetros do suporte ventilatório de pacientes com COVID-19, bem como os critérios e métodos utilizados para o desmame da ventilação mecânica invasiva, a partir de análise da produção científica atual sobre o tema.

\section{REVISÃO BIBLIOGRÁFICA}

A COVID-19 é uma síndrome infectocontagiosa do trato respiratório, causada pelo novo coronavírus (SARS-CoV-2), descrito como o causador de uma pandemia de pneumonia viral, tendo como o epicentro a cidade de Wuhan, na China, no final de 2019. O vírus pertence à família Coronovirinae (CoV), conhecida por causar infecções respiratórias em seres humanos e já foi responsável por duas grandes epidemias: a Síndrome Respiratória Aguda Grave (SARS-CoV) e a Síndrome Respiratória do Oriente Médio (MERS-CoV) (LU H, et al., 2020).

O SARS-CoV-2, são vírus de RNA de fita simples, com estrutura envelopada, capacitados a realizar mutações pontuais e recombinações genéticas, que originam o desenvolvimento de cepas com maior poder de patogenicidade, oferecendo um maior risco a população. O surgimento de mutações é um evento natural e esperado dentro do processo evolutivo dos vírus (OLIVEIRA TF, et al., 2021).

O SARS-CoV-2 possuem a chamada proteína $S$, e mutações nessa proteína são as mais relevantes do ponto de vista clínico e epidemiológico, uma vez que esta desempenha um papel importante durante o processo infeccioso facilitando a entrada do coronavírus nas células humanas. Recentemente, foi documentado que pessoas infectadas com a variante VOC 202012/01 têm um risco maior de óbito do que as pessoas infectadas com outras variantes. E estudos preliminares sugerem, ainda, que a variante 501Y.V2 está associada a uma carga viral mais alta, o que pode sugerir um potencial de maior transmissibilidade (OMS, 2021).

Hipóteses atuais sugerem que a origem da transmissão do SARS-CoV-2 para a espécie humana tenha sido a partir do contato direto com alimentos em comércio de frutos do mar, e também advindos da venda de animais silvestres vivos, como coelhos, pangolins, morcegos e répteis (WU F, et al., 2020). Por ser definida como uma doença infectocontagiosa, com predomínio do trato respiratório, a transmissão do patógeno pode ocorrer de forma direta entre indivíduos ou através da inalação de secreções respiratórias advindas de tosse, fala e espirro. A transmissão fecal-oral e a forma indireta por superfícies de contato contaminadas também contribuem para perdurar a ação viral (ZHANG W, et al., 2020).

A fisiopatologia da COVID-19 não está totalmente esclarecida. No entanto, foi confirmado que o mecanismo de entrada do vírus na célula hospedeira, exige a interação entre a sua proteína $S$ e os receptores de enzima conversora de angiotensina 2 (ECA2) presentes na superfície de diversos tipos celulares da espécie humana. Tais evidências, ajudam explicar a diversidade de manifestações clínicas associadas à infecção pelo SARS-CoV-2, devido a expressão da ACE2 nas células do trato respiratório, células tubulares renais, artérias, células gástricas e intestinais (ZHOU P, et al., 2020).

O período de incubação após o contágio pode variar de dois a 14 dias sendo, na maioria dos casos, em aproximadamente cinco dias após a exposição inicial. Decorrido este período, os indivíduos contaminados podem apresentar um amplo espectro clínico, desde uma infecção assintomática ou doença leve do trato respiratório inferior até uma pneumonia grave com insuficiência respiratória e falência de múltiplos órgãos, podendo levar ao óbito (ARAÚJO MS, et al., 2021). As complicações da COVID-19 e as taxas de mortalidade estão relacionadas com uma maior prevalência em pacientes do sexo masculino, com idade acima de 60 anos, estados de imunossupressão e a presença de comorbidades como cardiopatias, hipertensão, obesidade, diabetes, doenças respiratórias crônicas e neoplasias (GUAN W, et al., 2020).

Nos estados graves da infecção pelo novo coronavírus, destaca-se à síndrome do desconforto respiratório agudo (SDRA), na qual o desenvolvimento da insuficiência respiratória pelo paciente é devido à destruição 
do parênquima pulmonar, com intensa consolidação, associado à inflamação intersticial, culminando em uma oxigenação inadequada. Esse estágio crítico da doença, pode ser devido a uma "tempestade de citocinas pró-inflamatórias", onde o organismo desencadeia uma resposta inflamatória aumentada, com o objetivo de erradicar o SARS-CoV-2 (NISHIURA H, et al., 2020; BRITO SP, et al., 2020).

Devido ao aumento expressivo dos casos graves pela infecção por COVID-19, houve redução do número disponível de leitos de Unidades de Tratamento Intensivo (UTI), obrigando as instituições formularem protocolos que visem hierarquizar a admissão desses pacientes nessas unidades. Dentre os critérios utilizados para internação estão: (1) pacientes com necessidade de oxigênio suplementar para manutenção de saturação periférica de oxigênio $\left(\mathrm{SpO}_{2}\right)>94 \%$; (2) necessidade de ventilação não invasiva para manter $\mathrm{SpO}_{2}>94 \%$; (3) quadro de insuficiência respiratória aguda com necessidade de ventilação mecânica invasiva; (4) paciente com instabilidade hemodinâmica, choque ou sepse com hipotensão arterial (CARRILLO-ESPER $R$, et al., 2020).

Dentre as diversas abordagens terapêuticas para o manejo de paciente com formas graves de COVID-19 em UTI, destacam-se a Ventilação Mecânica (VM), o uso de agentes de bloqueio neuromusculares, o uso da posição prona e a Oxigenação Extracorpórea por Membrana (ECMO) (NATANOV R, et al., 2021). Alguns autores indicam que a utilização de Ventilação Não Invasiva (VNI) em modalidades de pressão positiva em dois níveis e o uso do cateter nasal de alto fluxo não são recomendados no tratamento das formas graves em decorrência da grande produção de aerossóis, o que culmina em maiores chances de contaminação da equipe e do ambiente, além de terem menor impacto na sobrevida frente aos tratamentos em destaque (CORRÊA T, et al., 2020).

No entanto, a crescente produção científica a respeito do tema trouxe novos parâmetros de utilização da VNI, sinalizando que sua utilização de forma precoce, em associação ou não à posição prona, reduziu a necessidade de intubação orotraqueal em inúmeros casos, sobretudo quando realizada por meio de capacetes (helmet) (WANG K, et al., 2020).

Estatísticas indicam que, aproximadamente, entre 250 mil e 1 milhão de pacientes com infecção pela COVID-19 precisaram de ventilação invasiva em todo o mundo. Além disso, a mortalidade dos pacientes com formas graves apresentou notável associação nos casos em que a ventilação mecânica mostrou-se necessária. Entretanto, mesmo diante da alta demanda da ventilação invasiva no tratamento da forma grave, o número de estudos publicados com foco a respeito da mecânica respiratória, configurações de ventilação mecânica e parâmetros de troca gasosa permanece escasso e ainda não há um consenso (GRASSELLI G, et al., 2021).

A literatura disponível mostra que pacientes gravemente enfermos com COVID-19 que requerem ventilação invasiva apresentam padrões heterogêneos de trocas gasosas e mecânica respiratória durante as primeiras 24 horas de admissão na UTI. Esse achado é caracteristicamente compatível com SDRA por outras causas (GRASSELLI G, et al., 2021).

Diante desta heterogeneidade, os parâmetros iniciais recomendados para a VM e mais comumente usados estão o modo ventilação com volume controlado (VCV) ou ventilação com pressão controlada (PCV) com volume corrente baixo em torno de $6 \mathrm{ml} / \mathrm{kg}$ de peso, sendo o peso em mulheres calculado como (altura em $\mathrm{cm}-152,4 \times 0,91)+45,5$ e nos homens como (altura em cm - 152,4) $\times 0,91+50$. Alto volume corrente no primeiro dia de ventilação foi associado a maior risco de mortalidade em 28 dias. Esse achado está de acordo com publicações anteriores em pacientes com SDRA por outras causas (CORRÊA T, et al., 2020; BOTTA M, et al., 2021).

A fração de inspiração de oxigênio $\left(\mathrm{FiO}_{2}\right)$ pode ser iniciada em $60 \%$, sendo titulada para alcançar um alvo de $\mathrm{SpO} 2$ entre $92 \%$ e $96 \%$. A frequência respiratória do ventilador deverá ser, preferencialmente, de até 20 respirações por minuto e a pressão de platô deve ser mantida abaixo de $30 \mathrm{~cm} \mathrm{H_{2 } O}$ para evitar a superdistensão alveolar (CAMPOS NG e COSTA RF, 2020; CARRILLO-ESPER R, et al., 2020). Uma pressão de platô mais alta sem o desenvolvimento de lesão pulmonar induzida por ventilador pode ser considerada nos pacientes com obesidade central (BERLIN DA, et al., 2020). 
Pacientes com COVID-19 ventilados mecanicamente possuem complacência pulmonar relativamente alta, comprometimento da troca gasosa e alta ventilação de espaço morto. Níveis elevados de pressão expiratória final positiva (PEEP) diminuem a complacência pulmonar e, na maioria dos casos, aumentam a ventilação do espaço morto, causando hiperinsulflação de partes mais complacentes do pulmão. Portanto, recomenda-se níveis mais baixos de PEEP tanto quanto possível. Para pacientes com SDRA relacionada a Covid-19, definir PEEP suficiente no ventilador pode prevenir o colapso alveolar e facilitar o recrutamento de regiões pulmonares instáveis (ROESTHUIS L, et al., 2020; BERLIN DA, et al., 2020).

A ventilação na Posição Prona (PP) é considerada tratamento adjuvante em pacientes gravemente enfermos, sendo recomendada atualmente por diversas diretrizes. O objetivo da pronação é aumentar o recrutamento alveolar, reduzir o shunt alveolar e melhorar a relação ventilação/perfusão, corrigindo posteriormente a hipoxemia e distribuindo a ventilação pulmonar de forma mais homogênea. Portanto, a PP melhora as trocas gasosas e a mecânica pulmonar, devendo ser considerada nos estágios iniciais da insuficiência respiratória em pacientes com SDRA grave por COVID-19, sendo que as evidências recentes sugerem que a sua aplicação precoce reduz a mortalidade (HERNÁNDEZ AV, et al., 2020; ARAUJO MS, et al., 2021).

Dentre os critérios utilizados pelas diretrizes para aplicar à $\mathrm{PP}$, estão a relação $\mathrm{PaO}_{2} / \mathrm{FIO}_{2}$, a $\mathrm{SpO}_{2}$ e a frequência respiratória. Recomenda-se um tempo longo de uso de protocolo de prona (16 a 20 horas/dia por 3 a 5 dias, por exemplo). Por fim, complicações da utilização da PP foram identificadas, sendo as mais relevantes a extubação acidental, lesão por pressão e edema facial. Entretanto, desfechos positivos sobressaem-se às complicações, sendo evidente a redução da hipoxemia e mortalidade (ARAUJO MS, et al., 2021; ROESTHUIS L, et al., 2020).

A utilização de ECMO ainda é pouco abordada na literatura. Seu uso é indicado para casos críticos, visando à proteção dos pulmões e à preservação das funções pulmonares. Seu circuito é composto por uma bomba de propulsão de sangue, cânulas de drenagem e retorno do sangue, oxigenador, sensores de fluxo, sensores de pressão, sistema de controle de temperatura e pontos de acesso arterial ou venoso. O sangue do paciente é impulsionado pela bomba, passando pelo oxigenador, um recipiente contendo duas câmaras, separadas por uma membrana de oxigenação. Ocorre, então, a difusão de gases, por meio da membrana, entre a câmara contendo o sangue do paciente e aquela contendo uma mistura gasosa denominada de fluxo do ar fresco, permitindo a oxigenação do sangue venoso (RABELLO R, et al., 2019).

A principal vantagem do uso de ECMO é o controle sobre a falência da função pulmonar, permitindo sua preservação. Comparando-se parâmetros observados em pacientes que sobreviveram após o uso de ECMO com aqueles de pacientes que faleceram, notaram-se algumas diferenças, tais como: níveis consideravelmente mais elevados de interleucina- 6 após o óbito, pior índice Horowitz, necessidade de maiores concentrações de noradrenalina, maiores concentrações de procalcitonina, maior nível de lactato desidogrenase e maior ocorrência de hemólise (NATANOV R, et al., 2021)

O desmame da ventilação mecânica, por sua vez, é um processo individualizado e é necessário alcançar um equilíbrio entre a carga e a capacidade do sistema respiratório para que se obtenha sucesso. Esse processo compreende a redução gradual dos parâmetros da ventilação mecânica e abrange diversos aspectos relacionados ao estado do paciente, como médicos, psicológicos, físicos e nutricionais (MCCONVILLE JF e KRESS JP, 2013; OVADYA D, et al., 2020). Consideram-se adequados para pacientes com COVID-19 os critérios tradicionais de extubação. Os pacientes considerados elegíveis para um teste de respiração espontânea devem receber fisioterapia respiratória antes e após a realização da extubação, uma vez que foram observados melhores resultados nos pacientes que passaram por esse processo (LAZZERI M, et al., 2020; THOMAS P, et al., 2020).

A retirada da ventilação mecânica não deve ser acelerada, mas sim gradual, com uma despressurização lenta do sistema ventilatório através de uma redução gradativa do nível de PEEP e verificação se as metas de oxigenação foram alcançadas, sendo tolerado um atraso de até duas semanas devido a evolução lenta desses pacientes. A retirada precoce da ventilação mecânica pode acarretar em uma maior deterioração e piorar o prognóstico dos pacientes (CARRILLO-ESPER R, et al., 2020). 
Deve-se considerar ventilação mecânica não-invasiva, pressão positiva contínua nas vias aéreas e oxigênio nasal de alto fluxo pouco tempo após a extubação, até que o paciente alcance a autonomia respiratória completa. Além disso, a traqueostomia é um procedimento que pode ser utilizado em pacientes em estado crítico, com o propósito de facilitar o desmame da ventilação mecânica e a higiene das vias aéreas, além de reduzir o risco de complicações associadas à intubação prolongada (LAZZERI M, et al., 2020; BOTTI C, et al., 2020).

Em pacientes pediátricos, a extubação também é um momento bastante crítico, sendo mais frequente a extubação não planejada, o que requer muita atenção da equipe médica. Recomenda-se atingir níveis de ventilação bem baixos antes da extubação, como $\mathrm{FiO} 2<30 \%$, pressão de suporte $\leq 10 \mathrm{cmH} 2 \mathrm{O}$ e Pressão Expiratória Final Positiva $\leq 7 \mathrm{cmH} 2 \mathrm{O}$. É importante que o reflexo do vômito e outros reflexos de proteção das vias aéreas sejam testados antes da extubação, uma vez que há relatos de casos nos quais os pacientes com aspiração ou secreções demasiadas das vias aéreas foram reintubados (CARVALHO WB, et al., 2020).

\section{CONSIDERAÇÕES FINAIS}

Diante do exposto, entende-se que a utilização de ventilação invasiva e internação em UTI pode ser necessária em casos mais graves da infecção por COVID-19, onde a ventilação com pressão controlada com baixo volume corrente está associada com melhor prognóstico. Além disso, devido à alta complacência pulmonar observada em pacientes com COVID-19 ventilados mecanicamente, recomenda-se níveis mais baixos de PEEP tanto quanto possível. O desmame ventilatório desses pacientes é um processo individualizado, guiado pelos aspectos médicos, físicos, nutricionais e psicológicos. A retirada da ventilação deve ser feita de forma lenta e gradual, observando a resposta do paciente em relação às metas de oxigenação e restabelecimento da autonomia respiratória.

\section{REFERÊNCIAS}

1. ARAÚJO MS, et al. Prone positioning as an emerging tool in the care provided to patients infected with COVID-19: a scoping review. Revista Latino-Americana de Enfermagem, 2021; 29: 3397.

2. AYRES, J. S. A metabolic handbook for the COVID-19 pandemic. Nature Metabolismo, 2020; 2: 572-585

3. BASTOS GAN, et al. Características clínicas e preditores de ventilação mecânica em pacientes com COVID-19 hospitalizados no sul do país. Revista Brasileira de Terapia Intensiva, 2020; 32(4): 487-492.

4. BERLIN DA, et al. Severe Covid-19. The New England Journal of Medicine, 2020; 383: 2451-2460.

5. BOTTA M, et al. Ventilation Management and Clinical Outcomes in Invasively Ventilated Patients with COVID-19 (PRoVENT-COVID): A National, Multicentre, Observational Cohort Study. The Lancet Respiratory Medicine, 2021; 9(2): 139-48.

6. BRITO SP, et al. Pandemia da COVID-19: O maior desafio do século XXI. Vigilância Sanitária Em Debate: Sociedade, Ciência \& Tecnologia - Revista Visa Em Debate, 2020; 8(2): 54-63.

7. CAMPOS NG, COSTA RF. Alterações pulmonares causadas pelo novo Coronavírus (COVID-19) e o uso da ventilação mecânica invasiva. Journal of Health \& Biological Sciences, 2020; 8(1): 1-3.

8. CARRILLO-ESPER, R, et al. Abordaje hemodinâmico y ventilatorio en pacientes con COVID-19. Cirurgía y Cirujanos, 2020; 88(6).

9. CARVALHO WB, et al. Ventilatory support recommendations in children with Sars-CoV-2. Revista da Associação Médica Brasileira, 2020; 66(4): 528-533

10. CORRÊA T, et al. Recomendações para suporte intensivo para pacientes graves com infecção suspeita ou confirmada pela COVID-19. Einstein (São Paulo), 2020; 18: 1-9.

11. GRASSELLI G, et al. Mechanical ventilation parameters in critically ill COVID-19 patients: a scoping review. Critical Care, 2021; 25: 115.

12. GUAN W, et al. Clinical characteristics of coronavirus disease 2019 in China. The New England Journal of Medicine, 2020; 382: 1-13.

13. HERNÁNDEZ AV, et al. Tratamiento de soporte respiratorio de la COVID-19. Papel de las unidades de cuidados respiratorios intermedios. Revista de patología respiratoria, 2020; 23 (3): 279-284.

14. LAZZERI M, et al. Respiratory physiotherapy in patients with COVID-19 infection in acute setting: a Position Paper of the Italian Association of Respiratory Physiotherapists (ARIR). Monaldi Archives for Chest Disease, 2020; 90(1).

15. LEWIS SR, et al. High-flow nasal cannulae for respiratory support in adult intensive care patients (Review). Cochrane Database of Systematic Reviews, 2021: CD010172.

16. LU H, et al. Outbreak of Pneumonia of Unknown Etiology in Wuhan, China: The Mystery and the Miracle. Journal of Medical Virology, 2020; 92(4): 401-402. 
17. MCCONVILLE JF, KRESS JP. Weaning patients from the ventilator. New England Journal of Medicine, 2012; 367(23): 2233-2239.

18. NATANOV R, et al. Mechanical circulatory support in coronavirus disease-2019-positive patients with severe respiratory failure. Interactive Cardiovascular and Thoracic Surgery, 2021; $1-5$.

19. NISHIURA H, et al. The extent of transmission of novel coronavirus in Wuhan, China, 2020. Journal of Clinical Medicine, 2020; 9(2): 330.

20. NOBRE K, et al. Treatment of patients with acute respiratory insufficiency due to COVID-19: invasive and non-invasive mechanical conditions. Journal of research and knowledge spreading, 2020; 1(1): e11672.

21. OLIVEIRA TF, et al. COVID-19: Revisão Narrativa. Revista Artigos.Com, 2021; 25: e4252.

22. ORGANIZAÇÃO MUNDIAL DA SAÚDE (OMS). WHO Coronavirus (COVID-19) Dashboard. 2021. Disponível em: https://covid19.who.int/. Acessado em: 26 de maio de 2021.

23. OVADYA D, et al. Weaning of severe COVID-19 Mechanically Ventilated Patients: Experience within a Dedicated Unit in Israel. The Israel Medical Association Journal, 2020; 22: 733-735.

24. RABELLO R, et al. Oxigenação por membrana extracorpórea: revisão da literatura. Rev. Bras. Ter. Intensiva, 2019; 31(3): 410-424.

25. ROESTHUIS L, et al. Advanced Respiratory Monitoring in COVID-19 Patients: Use Less PEEP!. Critical Care, 2020; 24(1): 230.

26. THOMAS P, et al. Physiotherapy management for COVID-19 in the acute hospital setting: clinical practice recommendations. Journal of Physiotherapy, 2020; 66(2): 73-82.

27. WANG K, et al. The experience of high-flow nasal cannula in hospitalized patients with 2019 novel coronavirusinfected pneumonia in two hospitals of Chongqing, China. Annals of Intensive Care, 2020; $10: 37$.

28. WU F, et al. A new coronavirus associated with human respiratory disease in China. Nature, 2020; 579(7798): 265269.

29. ZHANG W, et al. Molecular and serological investigation of 2019-nCoV infected patients: implication of multiple shedding routes. Emerging microbes \& infection, 2020; 9(1): 386-389.

30. ZHOU P, et al. A pneumonia outbreak associated with a new coronavirus of probable bat origin. Nature, 2020; 579(7798): 270-273. 